MATHEMATICS OF COMPUTATION

Volume 76, Number 258, April 2007, Pages 1059-1071

S 0025-5718(07)01926-6

Article electronically published on January 5, 2007

\title{
COMPUTATION OF THE $p$-PART \\ OF THE IDEAL CLASS GROUP OF CERTAIN REAL ABELIAN FIELDS
}

\author{
HIROKI SUMIDA-TAKAHASHI
}

\begin{abstract}
Under Greenberg's conjecture, we give an efficient method to compute the $p$-part of the ideal class group of certain real abelian fields by using cyclotomic units, Gauss sums and prime numbers. As numerical examples, we compute the $p$-part of the ideal class group of the maximal real subfield of $\mathbf{Q}\left(\sqrt{-f}, \zeta_{p^{n+1}}\right)$ in the range $1<f<200$ and $5 \leq p<100000$. In order to explain our method, we show an example whose ideal class group is not cyclic.
\end{abstract}

\section{INTRODUCTION}

Let $K$ be a number field and $p$ a prime number. Let $K_{\infty}$ be the cyclotomic $\mathbf{Z}_{p}$-extension of $K$ and $K_{n}$ the subfield of $K_{\infty}$ such that $\left[K_{n}: K\right]=p^{n}$. Further let $A_{n}$ be the $p$-part of the ideal class group of $K_{n}$. Greenberg's conjecture claims that $\sharp A_{n}$ is bounded as $n \rightarrow \infty$ if $K$ is totally real. We have not been able to find any counter-example to the conjecture. On the other hand, it has been verified for certain real abelian fields and some prime numbers by computer calculation (cf. [8, 16]).

In 11, 14, under Greenberg's conjecture, Kraft-Schoof and Ozaki gave a nice method to compute the $p$-part of the ideal class group of certain real abelian fields by using cyclotomic units. In the computation, we need to know whether a cyclotomic unit $c_{n} \in K_{n}$ is a $p^{n+1}$ th power or not in $K_{n}$. As the degree of the minimal polynomial for $c_{n}$ over $\mathbf{Q}$ gets larger, the computation of the minimal polynomial for $\sqrt[n+1]{c_{n}}$ becomes more difficult. In [15, 16, by using Gauss sums and prime numbers, we avoided the difficulty and gave an efficient method to compute the $p$ part of the ideal class number of certain real abelian fields. In this paper, combining them, we give an efficient method to compute the $p$-part of the ideal class group.

Following [15, 16, we give numerical examples of the $p$-part of the ideal class group of the maximal real subfield $K_{f, p}$ of $\mathbf{Q}\left(\sqrt{-f}, \zeta_{p}\right)$ in the range $1<f<200$ and $5 \leq p<100000$. The first purpose of this computation is to verify Greenberg's conjecture for each case. In fact we verify the conjecture in the above range. Therefore we can make use of the method of [11, 14 to compute the structure

Received by the editor September 7, 2005 and, in revised form, January 20, 2006.

2000 Mathematics Subject Classification. Primary 11R23, 11R70.

Key words and phrases. Ideal class group, Iwasawa invariant, abelian field, Greenberg's conjecture.

This work was partially supported by the Grants-in-Aid for Encouragement of Young Scientists (No. 16740019) from Japan Society for the Promotion of Science.

(C)2007 American Mathematical Society Reverts to public domain 28 years from publication 
of the $p$-part of the ideal class group. Let $\chi$ be the nontrivial Dirichlet character associated to $\mathbf{Q}(\sqrt{-f})$ and $\omega=\omega_{p}$ the Teichmüller character. Here we call $\left(p, \chi \omega^{k}\right)$ an exceptional pair if and only if $\chi \omega^{k}$ is even, $\chi \omega^{k}(p) \neq 1, \chi \omega^{1-k}(p) \neq 1$, and one of the following conditions is satisfied: $\nu_{p}\left(\chi \omega^{k}\right)>0, v_{p}\left(L_{p}\left(1, \chi \omega^{k}\right)\right)>$ $1, v_{p}\left(L_{p}\left(0, \chi \omega^{k}\right)\right)>1$, or $\tilde{\lambda}_{p}\left(\chi \omega^{k}\right)>1$, where $\nu_{p}\left(\chi \omega^{k}\right)$ is the $\chi \omega^{k}$-part of the Iwasawa $\nu_{p}$-invariant, $v_{p}$ is the $p$-adic valuation such that $v_{p}(p)=1$ and $\tilde{\lambda}_{p}\left(\chi \omega_{k}\right)$ is the degree of the Iwasawa polynomial for $\chi \omega^{k}$. The second purpose of the computation is to find exceptional pairs, as many as possible for large prime numbers in order to argue about their expected numbers (cf. [17, pp.158-159]). From our data, the actual numbers of exceptional pairs seem to be close to the expected numbers.

Following [1, we compute $A_{n}$ for $f=4 \cdot 14606$ and $p=5$ (i.e., $K=K_{4 \cdot 14606,5}$ ):

$$
\left\{\begin{array}{l}
A_{0} \simeq \mathbf{Z} / p \mathbf{Z} \oplus \mathbf{Z} / p \mathbf{Z}, \\
A_{n} \simeq \mathbf{Z} / p^{2} \mathbf{Z} \oplus \mathbf{Z} / p \mathbf{Z} \text { for } n \geq 1 .
\end{array}\right.
$$

Since $p$ splits in $\mathbf{Q}(\sqrt{-f})$, we need to modify some conditions in order to apply the criterion of 15 . We explain about the modification and difficulty in the following section.

\section{A method of Computation of $A_{n}$}

Let $F$ be an abelian field and $p$ an odd prime number. For simplicity, we assume the following condition:

$$
\text { The exponent of } \operatorname{Gal}(F / \mathbf{Q}) \text { divides } p-1 \text {. }
$$

Let $K=F\left(\zeta_{p}\right)$ and $A_{n}=A_{n}(K)$ be the $p$-part of the ideal class group of $K_{n}=$ $F\left(\zeta_{p^{n+1}}\right)$. Let $D_{n}$ be the subgroup of $A_{n}$ consisting of classes which contain an ideal all of whose prime factors lie above $p$. Set $A_{n}^{\prime}=A_{n} / D_{n}$. Let $M_{n}$ be the maximal abelian extension of $K_{n}$ unramified outside $p, L_{n}$ the maximal unramified abelian extension of $K_{n}$, and $L_{n}^{\prime}$ the maximal unramified abelian extension of $K_{n}$ in which every prime divisor above $p$ splits completely. Set $X_{n}=\operatorname{Gal}\left(L_{n} / K_{n}\right)$ and $X_{n}^{\prime}=\operatorname{Gal}\left(L_{n}^{\prime} / K_{n}\right)$. By the class field theory, we have $A_{n} \simeq X_{n}$ and $A_{n}^{\prime} \simeq X_{n}^{\prime}$. Set $L_{\infty}=\bigcup L_{n}, L_{\infty}^{\prime}=\bigcup L_{n}^{\prime}, X_{\infty}=\operatorname{Gal}\left(L_{\infty} / K_{\infty}\right)$ and $X_{\infty}^{\prime}=\operatorname{Gal}\left(L_{\infty}^{\prime} / K_{\infty}\right)$.

Set $\Delta=\operatorname{Gal}\left(K_{\infty} / \mathbf{Q}_{\infty}\right) \simeq \operatorname{Gal}\left(K_{0} / \mathbf{Q}\right)$. Let $\psi$ be a Dirichlet character of $\Delta$ and $e_{\psi}=\frac{1}{\sharp \Delta} \sum_{\delta \in \Delta} \psi(\delta) \delta^{-1} \in \mathbf{Z}_{p}[\Delta]$. For a $\mathbf{Z}_{p}[\Delta]$-module $A$, we denote $e_{\psi} A$ by $A^{\psi}$. Let $\lambda_{p}(\psi), \mu_{p}(\psi)$ and $\nu_{p}(\psi)$ (resp. $\lambda_{p}^{\prime}(\psi), \mu_{p}^{\prime}(\psi)$ and $\left.\nu_{p}^{\prime}(\psi)\right)$ be the Iwasawa invariants associated to $A_{n}^{\psi}$ (resp. $\left.A_{n}^{\prime}{ }_{n}\right)$, i.e.,

$$
\sharp A_{n}^{\psi}=p^{\lambda_{p}(\psi) n+\mu_{p}(\psi) p^{n}+\nu_{p}(\psi)} \quad\left(\text { resp. } \sharp A_{n}^{\prime \psi}=p^{\lambda_{p}^{\prime}(\psi) n+\mu_{p}^{\prime}(\psi) p^{n}+\nu_{p}^{\prime}(\psi)}\right)
$$

for all sufficiently large integers $n$. By Ferrero-Washington's theorem in [4, we have $\mu_{p}(\psi)=\mu_{p}^{\prime}(\psi)=0$ for all $p$ and $\psi$.

Assume that $\psi$ is even. The Iwasawa polynomial $g_{\psi}(T) \in \mathbf{Z}_{p}[T]$ for the $p$-adic $L$-function is defined as follows. Let $L_{p}(s, \psi)$ be the $p$-adic $L$-function constructed in [12. Let $f_{0}$ be the least common multiple of $p$ and $f_{\psi}$ the conductor of $\psi$. By [9, $\S 6]$, there uniquely exists $G_{\psi}(T) \in \mathbf{Z}_{p}[[T]]$ satisfying $G_{\psi}\left(\left(1+f_{0}\right)^{1-s}-1\right)=L_{p}(s, \psi)$ for all $s \in \mathbf{Z}_{p}$ if $\psi \neq \psi^{0}$. By [4, it was proved that $p$ does not divide $G_{\psi}(T)$. Therefore, by the $p$-adic Weierstrass preparation theorem, we can uniquely write $G_{\psi}(T)=g_{\psi}(T) u_{\psi}(T)$, where $g_{\psi}(T)$ is a distinguished polynomial of $\mathbf{Z}_{p}[T]$ and $u_{\psi}(T)$ is an invertible element of $\mathbf{Z}_{p}[[T]]$. Similarly we can define $g_{\psi}^{*}(T) \in \mathbf{Z}_{p}[T]$ 
from $G_{\psi}^{*}(T) \in \mathbf{Z}_{p}[[T]]$ satisfying $G_{\psi}^{*}\left(\left(1+f_{0}\right)^{s}-1\right)=L_{p}(s, \psi)$. Put $\tilde{\lambda}_{p}(\psi)=$ $\operatorname{deg} g_{\psi}(T)=\operatorname{deg} g_{\psi}^{*}(T)$.

Let $\gamma \in \Gamma=\operatorname{Gal}\left(\bigcup \mathbf{Q}\left(\zeta_{f_{n}}\right) / \mathbf{Q}\left(\zeta_{f_{0}}\right)\right) \simeq \operatorname{Gal}\left(K_{\infty} / K_{0}\right)$ be a generator of $\Gamma$ such that $\zeta_{f_{n}}^{\gamma}=\zeta_{f_{n}}^{1+f_{0}}$ for all $n \geq 0$ and $f_{n}=f_{0} p^{n}$. As usual, we can identify the complete group ring $\mathbf{Z}_{p}[[\Gamma]]$ with the formal power series ring $\Lambda=\mathbf{Z}_{p}[[T]]$ by $\gamma=1+T$. By this identification, we can consider a $\mathbf{Z}_{p}[[\Gamma]]$-module as a $\Lambda$-module. Set $\omega_{n}=$ $(1+T)^{p^{n}}-1$ and $\nu_{m, n}=\omega_{m} / \omega_{n}$ for $m \geq n \geq 0$. For a finitely generated torsion $\Lambda$-module $A$, we define the Iwasawa polynomial $\operatorname{char}_{\Lambda}(A)$ to be the characteristic polynomial of the action $T$ on $A \otimes \mathbf{Q}_{p}$ (cf. [17, §13]). By Mazur-Wiles' theorem in [13], $\operatorname{char}_{\Lambda}\left(X^{\psi^{-1} \omega}\right)=g_{\psi}^{*}(T)$.

Let $\mathfrak{p}$ be a prime ideal of $K$ over $p$ and $\mathfrak{p}_{n}$ the unique prime ideal of $K_{n}$ over $\mathfrak{p}$. Denote by $K_{\mathfrak{p}_{n}}$ the completion of $K_{n}$ at $\mathfrak{p}_{n}$, and by $\mathcal{U}_{\mathfrak{p}_{n}}$ the group of principal units of $K_{\mathfrak{p}_{n}}$. Put $\mathcal{V}_{\mathfrak{p}_{n}}=\bigcap_{m \geq n} N_{m, n} \mathcal{U}_{\mathfrak{p}_{n}}, N_{m, n}$ denoting the norm map from $K_{\mathfrak{p}_{m}}$ to $K_{\mathfrak{p}_{n}}$. We set

$$
\mathcal{U}_{n}=\prod_{\mathfrak{p} \mid p} \mathcal{U}_{\mathfrak{p}_{n}} \text { and } \mathcal{V}_{n}=\prod_{\mathfrak{p} \mid p} \mathcal{V}_{\mathfrak{p}_{n}},
$$

where $\mathfrak{p}$ runs over all prime ideals of $K$ over $p$.

Let $E_{n}^{\prime}$ be the group of units $\varepsilon$ of $K_{n}$ satisfying $\varepsilon \equiv 1 \bmod \mathfrak{p}_{n}$ for all $\mathfrak{p}_{n} \mid p$. Denote by $C_{n}$ the subgroup of $K_{n}^{\times}$generated by all the units

$$
N_{\mathbf{Q}\left(\zeta_{f_{n}}\right) / K_{n}}\left(1-\zeta_{f_{n}}\right)^{u}, \quad u \in \mathbf{Z}\left[\operatorname{Gal}\left(K_{n} / \mathbf{Q}\right)\right]^{0},
$$

where $X^{0}$ is the augmentation ideal of the group ring $X$. Denote, respectively, $\mathcal{E}_{n}$ and $\mathcal{C}_{n}$ as the closures of the images of $E_{n}^{\prime}$ and $C_{n}^{\prime}=C_{n} \cap E_{n}^{\prime}$ under the diagonal embedding $d_{n}: E_{n}^{\prime} \rightarrow \mathcal{U}_{n}$.

From now on, we also assume the following condition:

$$
\psi(p) \neq 1 \text {. }
$$

Set $\psi^{*}=\psi^{-1} \omega$ and $\omega_{0}^{*}=T-f_{0}$. Then we have the following facts (see [5, Theorem 1, 2]):

\section{Fact 1.}

$$
\mathcal{U}_{n}^{\psi}=\mathcal{V}_{n}^{\psi}
$$

If $\psi^{*}(p) \neq 1$,

If $\psi^{*}(p)=1$,

$$
\begin{array}{ccc}
\mathcal{U}_{n}^{\psi} & \simeq & \Lambda /\left(\omega_{n}\right) \\
\cup & \cup & \cup \\
\mathcal{C}_{n}^{\psi} & \simeq & \left(g_{\psi}(T), \omega_{n}\right) /\left(\omega_{n}\right) .
\end{array}
$$

$$
\begin{array}{rlc}
\mathcal{U}_{n}^{\psi} / \mathbb{T}_{n} & \simeq \quad & \Lambda /\left(\omega_{n}\right) \\
\cup & \cup \\
\mathcal{C}_{n}^{\psi} \mathbb{T}_{n} / \mathbb{T}_{n} & \simeq & \left(\tilde{g}_{\psi}(T), \omega_{n}\right) /\left(\omega_{n}\right),
\end{array}
$$

where $\mathbb{T}_{n}=\operatorname{Tor}_{\mathbf{z}_{p}} \mathcal{U}_{n}^{\psi} \simeq \Lambda /\left(\omega_{0}^{*}, \omega_{n}\right)$ and $\tilde{g}_{\psi}(T)=g_{\psi}(T) / \omega_{0}^{*}$.

We also have the following fact on $E_{n}^{\prime}$ and $\mathcal{E}_{n}$, which follows from the Leopoldt conjecture for $\left(K_{n}, p\right)$ (cf. [17, §5.5]):

Fact 2. The inclusion $d_{n}: E_{n}^{\prime} \rightarrow \mathcal{E}_{n}$ induces an isomorphism

$$
\left(E_{n}^{\prime} / E_{n}^{\prime p^{a}}\right)^{\psi} \simeq\left(\mathcal{E}_{n} / \mathcal{E}_{n}{ }^{p^{a}}\right)^{\psi}
$$

for any $a \geq 0$. Therefore $\mathcal{E}_{n}^{\psi}$ has no nontrivial torsion element. 
Our computation is based on the following theorem:

Theorem 1 (Kraft-Schoof, Ozaki). Assume (C1) and (C2). Greenberg's conjecture holds for $A_{n}^{\psi}$ if and only if there exists an integer $n_{0}$ such that $\left(\mathcal{E}_{n} / \mathcal{C}_{n}\right)^{\psi}$ is stabilized for $n \geq n_{0}$. Then

$$
A_{n}^{\psi} \simeq\left(\mathcal{E}_{n} / \mathcal{C}_{n}\right)^{\psi}
$$

for all $n \geq n_{0}$.

We give an outline of a proof for convenience of the readers.

Proof. By (C1) and (C2), we obtain the following exact sequence for $m \geq n$ (cf. [15, $\S 2]):$

$$
0 \rightarrow H^{1}\left(\Gamma_{n}, E_{m}\right)^{\psi} \rightarrow A_{n}^{\psi} \rightarrow\left(A_{m}^{\Gamma_{n}}\right)^{\psi} \rightarrow H^{2}\left(\Gamma_{n}, E_{m}\right)^{\psi} \rightarrow 0,
$$

where $E_{n}$ is the group of units of $K_{n}$ and $\Gamma_{n}=\Gamma^{p^{n}}$. If Greenberg's conjecture holds for $A_{n}^{\psi}$, we can take $m$ and $n(m \geq n)$ such that $N_{m, n}: A_{m}^{\psi} \simeq A_{n}^{\psi}$ and that $i_{n, m}$ : $A_{n}^{\psi} \rightarrow\left(A_{m}^{\Gamma_{n}}\right)^{\psi}$ is a zero map, where $i_{n, m}$ is the induced map by the natural inclusion $k_{n} \hookrightarrow k_{m}$ (see [6. Proposition 1]). Since we have $H^{2}\left(\Gamma_{n}, E_{m}\right)^{\psi} \simeq\left(E_{n} / N_{m, n} E_{m}\right)^{\psi}$,

$$
0 \rightarrow A_{m}^{\psi} \rightarrow\left(E_{n} / N_{m, n} E_{m}\right)^{\psi} \rightarrow 0 .
$$

Further, we have $C_{n}=N_{m, n} C_{m} \subseteq N_{m, n} E_{m}$ and $\sharp A_{m}^{\psi}=\sharp\left(\left(E_{m} / C_{m}\right)(p)\right)^{\psi}=$ $\sharp\left(\left(E_{n} / C_{n}\right)(p)\right)^{\psi}$ for $n \geq n_{0}$ by Mazur-Wiles' theorem, where $A(p)$ is the $p$-part of the finite abelian group $A$. Therefore we have $A_{n}^{\psi} \simeq A_{m}^{\psi} \simeq\left(\left(E_{n} / C_{n}\right)(p)\right)^{\psi} \simeq$ $\left(\left(E_{m} / C_{m}\right)(p)\right)^{\psi} \simeq\left(\mathcal{E}_{m} / \mathcal{C}_{m}\right)^{\psi}$.

Let $L_{n}\left(\psi^{*}\right)$ be the fixed subfield of $L_{n}$ by $\bigoplus_{\chi \neq \psi^{*}} X_{n}^{\chi}$. In a similar way, we define $M_{n}\left(\psi^{*}\right), L_{\infty}\left(\psi^{*}\right)$, etc. For an ideal $\mathfrak{L}$ of $K_{n}$, set $\sigma_{\mathfrak{L}}^{\psi^{*}}=\left(\frac{L_{n}\left(\psi^{*}\right) / K_{n}}{\mathfrak{L}}\right) \in$ $\left(X_{n} / \bigoplus_{\chi \neq \psi^{*}} X_{n}^{\chi}\right) \simeq X_{n}^{\psi^{*}}$, where $\left(\frac{*}{*}\right)$ is the Artin symbol. In order to calculate $\left(\mathcal{E}_{n} / \mathcal{C}_{n}\right)^{\psi}$, we use the following lemma:

Lemma 1. For $k \leq n+1$, if $c_{n} \in C_{n}^{\prime}$ satisfies

$$
d_{n}\left(c_{n}\right) \in\left(\mathcal{U}_{n}^{p^{k}}\right)^{\psi} \mathcal{C}_{n}^{p^{k}},
$$

then $\sqrt[p^{k}]{c_{n}} \in L_{n}\left(\psi^{*}\right)$. Further assume that

$$
X_{n}^{\psi^{*}} \text { is generated by } \sigma_{\mathfrak{L}_{i}}^{\psi^{*}} \text { for } \mathfrak{L}_{i} \nmid p \text { and } 1 \leq i \leq r .
$$

Then

$$
c_{n} \in E_{n}^{\prime p^{k}} \text { if and only if }\left(c_{n} \bmod \mathfrak{L}_{i}\right)_{1 \leq i \leq r} \in \prod_{i=1}^{r}\left(\mathcal{O}_{K_{n}} / \mathfrak{L}_{i}\right)^{p^{k}} .
$$

Proof. Since $\sqrt[p k]{c_{n}} \in M_{n}\left(\psi^{*}\right),(\mathrm{A})$ implies the former assertion. By (B), $K_{n}\left(\sqrt[p]{c_{n}}\right)$ $=K_{n}$ if and only if the splitting field of $\mathfrak{L}_{i}$ in $L_{n}\left(\psi^{*}\right) / K_{n}$ includes $K_{n}\left(\sqrt[p]{c_{n}}\right)$ for every $i$, i.e., $c_{n}$ is a $p^{k}$ th power at $\mathfrak{L}_{i}$. Therefore we obtain the latter assertion.

In [15, when $\psi^{*}(p) \neq 1$, we gave explicit conditions for (A) and (B) by using cyclotomic units, Gauss sums and prime numbers. When $\psi^{*}(p)=1, \omega_{0}$ divides $g_{\psi}^{*}(T)$. For $n=0$, we can obtain full information from Gauss sums of a subfield of $K_{0}$ (cf. [1]). However, for $n \geq 1$, we cannot directly obtain full information on $A_{n}^{\psi^{*}}$ from Gauss sums (see [7, §4] and the last example of section 3). So we will replace the conditions (A) and (B) with $(\tilde{\mathrm{A}})$ and $(\tilde{\mathrm{B}})$ in Lemma 4, 
Let us write the Kummer pairing:

$$
X_{\infty}^{\psi^{*}} \times W_{\infty}^{\psi} \rightarrow \mu_{p^{\infty}}=\bigcup\left\langle\zeta_{p^{n}}\right\rangle
$$

where $W_{\infty}$ is the subgroup of $K_{\infty}^{\times} \otimes \mathbf{Q}_{p} / \mathbf{Z}_{p}$ which corresponds to $X_{\infty}$ via Kummer theory. Let $\operatorname{Ker}_{X_{\infty}^{\psi^{*}}} \omega_{0}$ be the subgroup of $X_{\infty}^{\psi^{*}}$ consisting of all elements annihilated by $\omega_{0}$. Set $\tilde{X}_{\infty}^{\psi^{*}}=X_{\infty}^{\psi^{*}} / \operatorname{Ker}_{X_{\infty}^{*}} \omega_{0}$. By Ferrero-Greenberg's theorem in [3], $\omega_{0}$ does not divide $\tilde{g}_{\chi}^{*}(T)=g_{\psi}^{*}(T) / \omega_{0}$. Hence we have

$$
\varphi: X_{\infty}^{\psi^{*}} \hookrightarrow \bigoplus_{i=1}^{s} \Lambda /\left(g_{i}^{*}(T)\right) \oplus \Lambda /\left(\omega_{0}\right)
$$

where $\prod_{i=1}^{s} g_{i}^{*}(T)=\tilde{g}_{\psi}^{*}(T)$. Let $\pi$ be the projection from $\bigoplus_{i=1}^{s} \Lambda /\left(g_{i}^{*}(T)\right) \oplus \Lambda /\left(\omega_{0}\right)$ to $\bigoplus_{i=1}^{s} \Lambda /\left(g_{i}^{*}(T)\right)$. Then $\tilde{X}_{\infty}^{\psi^{*}} \simeq\left(\varphi\left(X_{\infty}^{\psi^{*}}\right)\left(\Lambda /\left(\omega_{0}\right)\right)\right) /\left(\Lambda /\left(\omega_{0}\right)\right) \simeq \pi\left(\varphi\left(X_{\infty}^{\psi^{*}}\right)\right)$. Hence $\tilde{X}_{\infty}^{\psi^{*}}$ has no nontrivial finite submodule (cf. [10, Theorem 18]) and $\operatorname{char}_{\Lambda}\left(\tilde{X}_{\infty}^{\psi^{*}}\right)=$ $\tilde{g}_{\psi}^{*}(T)$. Set $\tilde{W}_{\infty}^{\psi}=\omega_{0}^{*} W_{\infty}^{\psi}$. Then we have the following Kummer pairing:

$$
\tilde{X}_{\infty}^{\psi^{*}} \times \tilde{W}_{\infty}^{\psi} \rightarrow \mu_{p^{\infty}} .
$$

When $\psi^{*}(p)=1$, we consider the above paring. In order to obtain elements in $\tilde{W}_{\infty}^{\psi}$ satisfying $(\tilde{\mathrm{A}})$ in Lemma 4, we use the following lemma:

Lemma 2. For integers $n$ and $k$, we set

$$
C_{n, k}=\left\langle\left[c_{n}\right] \in\left(C_{n}^{\prime} E_{n}^{\prime p^{k}} / E_{n}^{\prime p^{k}}\right)^{\psi} \mid d_{n}\left(c_{n}\right) \in\left(\mathcal{U}_{n}^{p^{k}} \mathbb{T}_{n}\right)^{\psi} \mathcal{C}_{n}^{p^{k}}\right\rangle
$$

Let a be the minimum integer such that $p^{a} \in\left(\omega_{0}^{*}, \tilde{g}_{\psi}(T)\right)$. Then

$$
p^{a} C_{n, k} \subseteq \omega_{0}^{*} C_{n, k} \subseteq C_{n, k} .
$$

Assume that Greenberg's conjecture holds for $A_{n}^{\psi}$. Then

$$
\bigcup_{n, k} C_{n, k}=\tilde{W}_{\infty}^{\psi}
$$

Proof. By Ferrero-Greenberg's theorem, $\omega_{0}^{*}$ does not divide $\tilde{g}_{\psi}(T)$. Therefore the minimum integer $a$ exists. Write $p^{a}=\omega_{0}^{*} a(T)+\tilde{g}_{\psi}(T) b(T)$ for $a(T), b(T) \in \Lambda$. Then we have $d_{n}\left(c_{n}\right)^{p^{a}}=d_{n}\left(c_{n}\right)^{\omega_{0}^{*} a(T)} d_{n}\left(c_{n}\right)^{\tilde{g}_{\psi}(T) b(T)} \in \mathcal{C}_{n}^{\omega_{0}^{*}} \mathcal{C}_{n}^{p^{k}} \mathbb{T}_{n}$. Since $\mathcal{C}_{n} \cap \mathbb{T}_{n}=$ $\{1\}$, we have $p^{a}\left[c_{n}\right] \in \omega_{0}^{*} C_{n, k}$. Since $\mathbb{T}_{n}=\mathbb{T}_{n+k}^{p^{k}}, d_{n+k}\left(c_{n}\right)$ is a $p^{k}$ th power in $\mathcal{U}_{n+k}$ for $\left[c_{n}\right] \in C_{n, k}$. Therefore we have $C_{n, k} \subseteq W_{\infty}^{\psi}$ and $p^{a} C_{n, k} \subseteq \tilde{W}_{\infty}^{\psi}$. Let

$$
C_{n, k}^{\prime}=\left\langle\left[c_{n}\right]^{\prime} \in\left(C_{n}^{\prime} / C_{n}^{\prime p^{k}}\right)^{\psi} \mid d_{n}\left(c_{n}\right) \in\left(\mathcal{U}_{n}^{p^{k}} \mathbb{T}_{n}\right)^{\psi} \mathcal{C}_{n}^{p^{k}}\right\rangle .
$$

By Fact 1, for sufficiently large integers $n, C_{n, k}^{\prime} \simeq\left(\mathbf{Z} / p^{k} \mathbf{Z}\right)^{\tilde{\lambda}(\psi)}$. If Greenberg's conjecture holds, $\sharp\left(\mathcal{E}_{n} / \mathcal{C}_{n}\right)^{\psi}$ is bounded. Hence $C_{n, k}$ has a subgroup which is isomorphic to $\left(\mathbf{Z} / p^{k-k^{\prime}} \mathbf{Z}\right)^{\tilde{\lambda}(\psi)}$, where $k^{\prime} \leq k$ is a constant integer. Further the natural map $i_{n, m}: C_{n, k} \rightarrow C_{m, k+m-n}\left(\left[c_{n}\right] \mapsto\left[c_{n}{ }^{p^{m-n}}\right]\right)$ is injective. Therefore $i_{n, m}\left(C_{n, k}\right) \subseteq$ $p^{a} C_{m, k+m-n}$ for sufficiently large integers $m$. Since $\bigcup C_{n, k} \simeq\left(\mathbf{Q}_{p} / \mathbf{Z}_{p}\right)^{\tilde{\lambda}(\psi)} \simeq \tilde{W}_{\infty}^{\psi}$, we have the equality. 
Let $\tilde{L}_{\infty}\left(\psi^{*}\right)$ be the fixed subfield of $L_{\infty}\left(\psi^{*}\right)$ by $\operatorname{Ker}_{X_{\infty} \psi^{*}} \omega_{0}$. Set $\tilde{L}_{n}\left(\psi^{*}\right)=$ $L_{n}\left(\psi^{*}\right) \cap \tilde{L}_{\infty}\left(\psi^{*}\right)$ and $\tilde{X}_{n}^{\psi^{*}}=\operatorname{Gal}\left(\tilde{L}_{n}\left(\psi^{*}\right) / K_{n}\right) \simeq \tilde{A}_{n}^{\psi^{*}}$. Then we can write

$$
\tilde{X}_{n}^{\psi^{*}} \simeq \tilde{X}_{\infty}^{\psi^{*}} / \nu_{n, 0} \tilde{Y}_{\infty}^{\psi^{*}}
$$

for a submodule $\tilde{Y}_{\infty}^{\psi^{*}}$ of $\tilde{X}_{\infty}^{\psi^{*}}$ (see [17, Lemma 13.15]).

Let $\mathfrak{m}=(p, T)$ be the maximal ideal of $\Lambda$. In order to find ideals $\mathfrak{L}_{i}$ satisfying $(\tilde{B})$ in Lemma 4, we use the following lemma.

Lemma 3. Let $X$ be a finitely generated torsion $\Lambda$-module which has no nontrivial finite $\Lambda$-submodule. Assume that $\omega_{0}$ does not divide $\operatorname{char}_{\Lambda}(X)$. Then for any $\Lambda$ submodules $X^{\prime}$ and $Z$ of $X$ such that $Z \subseteq \mathfrak{m} \omega_{0} X,\left(\omega_{0} X+Z\right) / Z=\left(\omega_{0} X^{\prime}+Z\right) / Z$ holds if and only if $X=X^{\prime}$.

Proof. Since $\mathfrak{m}\left(\omega_{0} X\right) \supseteq Z$, we have $\omega_{0} X=\omega_{0} X^{\prime}$ by Nakayama's lemma. For any element $x \in X$, there exists $x^{\prime} \in X^{\prime}$ such that $\omega_{0} x=\omega_{0} x^{\prime}$. Since $\omega_{0}: X \rightarrow X(x \mapsto$ $\left.\omega_{0} x\right)$ is injective, we have $x=x^{\prime}$ and $X=X^{\prime}$.

In order to calculate $\left(\mathcal{E}_{n} / \mathcal{C}_{n}\right)^{\psi}$ for $\psi^{*}(p)=1$, we use the following lemma, which can be proved in a similar way to Lemma 1 :

Lemma 4. Assume that Greenberg's conjecture holds for $A_{n}^{\psi}$. For $k \leq n+1$, if $c_{n} \in C_{n}^{\prime}$ satisfies

$$
d_{n}\left(c_{n}\right) \in\left(\mathcal{U}_{n}^{p^{k}} \mathbb{T}_{n}\right)^{\psi} \mathcal{C}_{n}^{p^{k}},
$$

then $\sqrt[p k]{c_{n}} \in \tilde{L}_{n^{\prime}}\left(\psi^{*}\right)$ for some $n^{\prime}$. Further assume that

$$
\tilde{X}_{n^{\prime}}^{\psi^{*}} \text { is generated by } \tilde{\sigma}_{\mathfrak{L}_{i}}^{\psi^{*}} \text { for } \mathfrak{L}_{i} \nmid p \text { and } 1 \leq i \leq r \text {. }
$$

Then

$$
c_{n} \in E_{n}^{\prime p^{k}} \text { if and only if }\left(c_{n} \bmod \mathfrak{L}_{i}\right)_{1 \leq i \leq r} \in \prod_{i=1}^{r}\left(\mathcal{O}_{K_{n^{\prime}}} / \mathfrak{L}_{i}\right)^{p^{k}} .
$$

By Mazur-Wiles' theorem, we can obtain $\sharp A_{n}^{\psi^{*}}=\sharp X_{n}^{\psi^{*}}=\sharp\left(X_{\infty}^{\psi^{*}} / \nu_{n, 0} Y_{\infty}^{\psi^{*}}\right)$ from generalized Bernoulli numbers. However it is difficult to compute $\sharp \tilde{X}_{n}^{\psi^{*}}$ because it is difficult to determine $\tilde{Y}_{\infty}^{\psi^{*}}$ in $\tilde{X}_{\infty}^{\psi^{*}}$. Therefore, in general, we have a difficulty in checking $(\tilde{\mathrm{B}})$ by our method.

\section{NUMERICAL EXAMPLES OF IDEAL CLASS GROUPS}

Let $\chi$ be an odd primitive quadratic Dirichlet character, $f_{\chi}$ the conductor of $\chi$, and $p$ an odd prime number. Set $F=F_{\chi}=\mathbf{Q}\left(\sqrt{-f_{\chi}}\right)$ and $K=\mathbf{Q}\left(\sqrt{-f_{\chi}}, \zeta_{p}\right)$. Let $k$ be an odd integer with $3 \leq k \leq p-2$. Then $\chi \omega^{k}$ is an even character. For a pair $\left(p, \chi \omega^{k}\right)$, we set the following condition:

$$
\chi \omega^{k}(p) \neq 1 \text { and } \chi \omega^{1-k}(p) \neq 1 \text {. }
$$

If $\chi \omega^{k}(p) \neq 1$, we have $\lambda_{p}\left(\chi \omega^{k}\right)=\lambda_{p}^{\prime}\left(\chi \omega^{k}\right)$ and $\nu_{p}\left(\chi \omega^{k}\right)=\nu_{p}^{\prime}\left(\chi \omega^{k}\right)$. In the range $1<f_{\chi}<200,5 \leq p<100000$ and odd integers $k$ with $3 \leq k \leq p-2$, there are 14085400622 pairs of $\left(p, \chi \omega^{k}\right)$ satisfying (C). Among them, 296975 pairs satisfy $\tilde{\lambda}_{p}\left(\chi \omega^{k}\right)=1,43$ pairs $\tilde{\lambda}_{p}\left(\chi \omega^{k}\right)=2$, and two pairs $\tilde{\lambda}_{p}\left(\chi \omega^{k}\right)=3$. By the method of [8], we verified Greenberg's conjecture, i.e., $\lambda_{p}\left(\chi \omega^{k}\right)=0$ for each of them. Moreover, we checked $\nu_{p}\left(\chi \omega^{k}\right) \leq 2$ by the method of [16]. In the above range, 44 pairs do not satisfy $(\mathrm{C})$. For these cases, we also checked that $\lambda_{p}\left(\chi \omega^{k}\right)=$ 
$\nu_{p}\left(\chi \omega^{k}\right)=0$ by the method of $[8]$. Further, using the following lemma, we verified that $\lambda_{p}(\chi \omega)=\nu_{p}(\chi \omega)=0$ for all $f_{\chi}$ and $p$ in the above range.

Proposition 1. If $A_{0}^{\chi}$ is trivial, then $A_{n}^{\chi \omega}$ is trivial for every $n \geq 0$.

Proof. Assume that $A_{n}^{\chi \omega}$ is not trivial for some $n$. Then we have $\operatorname{deg} g_{\chi \omega}^{*}(T)=$ $\operatorname{deg} g_{\chi \omega}(T) \geq 1$. Hence, if $\chi(p) \neq 1$, we have $v_{p}\left(\sharp A_{0}^{\chi}\right)=v_{p}\left(g_{\chi \omega}^{*}(0)\right) \geq 1$. If $\chi(p)=1$, then $\chi \omega(p) \neq 1$. In this case, by the class field theory (see [8, Lemma 3]), $A_{n}^{\chi \omega} \neq\{0\}$ implies $A_{0}^{\chi \omega} \neq\{0\}$. Let $\mathfrak{a} \in c \in A_{0}^{\chi \omega}$ such that $\mathfrak{a}^{p}=(\alpha)$ for $\alpha \in K$. Further there exists $\varepsilon \in E_{0}^{\prime} \backslash E_{0}^{\prime p}$ such that $[\varepsilon] \in\left(E_{0}^{\prime} / E_{0}^{\prime p}\right)^{\chi \omega}$. Then we have $\sqrt[p]{\alpha}$, $\sqrt[p]{\varepsilon} \in M_{0}(\chi)$ and $\operatorname{Gal}(K(\sqrt[p]{\alpha}, \sqrt[p]{\varepsilon}) / K) \simeq \mathbf{Z} / p \mathbf{Z} \oplus \mathbf{Z} / p \mathbf{Z}$. Since $\left(\mathcal{U}_{0} / \mathcal{U}_{0}^{p}\right)^{\chi} \simeq \mathbf{Z} / p \mathbf{Z}$, there exists a nontrivial unramified abelian $p$-extension of $K$ contained in $M_{0}(\chi)$. Therefore, by the class field theory, $A_{0}^{\chi}$ is not trivial.

We obtain the following computational result:

Proposition 2. Let $K_{f_{\chi}, p}$ be the maximal real subfield of $\mathbf{Q}\left(\sqrt{-f_{\chi}}, \zeta_{p}\right) \cdot \lambda_{p}\left(K_{f_{\chi}, p}\right)$ $=0$ for all $1<f_{\chi}<200$ and $5 \leq p<100000$. Exactly,

$$
\begin{array}{lll}
A_{n}\left(K_{f_{\chi}, p}\right)=\{0\} & \text { for } n \geq 0 & \text { and }\left(f_{\chi}, p\right) \text { which does not appear in Table } 1, \\
A_{n}\left(K_{f_{\chi}, p}\right) \simeq \mathbf{Z} / p \mathbf{Z} & \text { for } n \geq 0 & \text { and }\left(f_{\chi}, p\right) \neq(136,11) \text { in Table } 1, \\
A_{n}\left(K_{f_{\chi}, p}\right) \simeq\left\{\begin{array}{lll}
\mathbf{Z} / p \mathbf{Z} & \text { for } n=0, & \text { and }\left(f_{\chi}, p\right)=(136,11) .
\end{array}\right.
\end{array}
$$

TABLE 1. $\nu_{p}\left(\chi \omega^{k}\right)=1$ (2 for the $*$-marked case $)$

\begin{tabular}{ccc|ccc|ccc|ccc}
\hline$f_{\chi}$ & $p$ & $k$ & $f_{\chi}$ & $p$ & $k$ & $f_{\chi}$ & $p$ & $k$ & $f_{\chi}$ & $p$ & $k$ \\
\hline 4 & 379 & 317 & 11 & 79 & 55 & 11 & 173 & 161 & 15 & 4909 & 2173 \\
19 & 37 & 17 & 19 & 41 & 11 & 19 & 2251 & 1953 & 20 & 20261 & 19403 \\
23 & 193 & 175 & 31 & 131 & 115 & 31 & 821 & 275 & 40 & 97 & 83 \\
51 & 557 & 457 & 51 & 6553 & 3593 & 55 & 41189 & 2099 & 67 & 433 & 409 \\
71 & 17 & 3 & 79 & 45943 & 18175 & 79 & 17 & 9 & 84 & 10133 & 9805 \\
88 & 33049 & 9069 & 91 & 37 & 21 & 91 & 7069 & 3293 & 103 & 17 & 3 \\
103 & 67 & 15 & 104 & 17837 & 285 & 116 & 4363 & 3845 & 120 & 4177 & 2253 \\
127 & 67 & 53 & 131 & 853 & 127 & 136 & 54547 & 6417 & 136 & 11 & $3 *$ \\
139 & 4451 & 2233 & 148 & 23 & 13 & 152 & 863 & 617 & 152 & 3019 & 2319 \\
155 & 12377 & 9137 & 163 & 79 & 55 & 167 & 797 & 245 & 187 & 79 & 63 \\
\hline
\end{tabular}

TABLE 2. $v_{p}\left(a_{0}\left(\chi \omega^{k}\right)\right)=2(3$ for the $*$-marked case $)$

\begin{tabular}{ccc|ccc|ccc|ccc}
\hline$f_{\chi}$ & $p$ & $k$ & $f_{\chi}$ & $p$ & $k$ & $f_{\chi}$ & $p$ & $k$ & $f_{\chi}$ & $p$ & $k$ \\
\hline 4 & 1381 & 609 & 11 & 17 & 7 & 15 & 31 & 5 & 19 & 2699 & 1579 \\
23 & 2521 & 2473 & 39 & 11 & 3 & 40 & 19 & 15 & 43 & 71 & 57 \\
47 & 373 & 53 & 52 & 83 & 79 & 52 & 241 & 51 & 79 & 7 & $5 *$ \\
79 & 41 & 5 & 79 & 4651 & 3373 & 84 & 31 & 25 & 88 & 70141 & 56107 \\
103 & 7 & 3 & 104 & 3637 & 1487 & 115 & 1381 & 357 & 116 & 11 & 3 \\
116 & 827 & 745 & 119 & 31 & 3 & 120 & 127 & 65 & 127 & 19 & 7 \\
131 & 37 & 19 & 131 & 251 & 61 & 131 & 16267 & 11043 & 131 & 39569 & 13871 \\
136 & 32869 & 6721 & 139 & 109 & 91 & 159 & 167 & 133 & 167 & 41 & 13 \\
168 & 11 & 3 & 168 & 1087 & 475 & 179 & 19 & 15 & 179 & 2161 & 1605 \\
187 & 17 & 15 & 199 & 19 & 9 & & & & & & \\
\hline
\end{tabular}


TABLE 3. $v_{p}\left(b_{0}\left(\chi \omega^{k}\right)\right)=2$

\begin{tabular}{ccc|ccc|ccc|ccc}
\hline$f_{\chi}$ & $p$ & $k$ & $f_{\chi}$ & $p$ & $k$ & $f_{\chi}$ & $p$ & $k$ & $f_{\chi}$ & $p$ & $k$ \\
\hline 3 & 257 & 101 & 7 & 173 & 97 & 19 & 52067 & 13617 & 19 & 71353 & 1597 \\
19 & 2711 & 41 & 20 & 193 & 27 & 39 & 1187 & 349 & 39 & 9007 & 7117 \\
43 & 757 & 123 & 51 & 107 & 27 & 71 & 46829 & 27893 & 71 & 1933 & 1275 \\
79 & 43 & 25 & 79 & 269 & 107 & 79 & 2417 & 1389 & 84 & 59 & 41 \\
88 & 19 & 11 & 91 & 277 & 99 & 91 & 1511 & 279 & 116 & 503 & 123 \\
119 & 23 & 19 & 120 & 107 & 31 & 120 & 421 & 9 & 123 & 19 & 11 \\
123 & 149 & 83 & 127 & 59183 & 29151 & 127 & 11 & 7 & 127 & 563 & 311 \\
127 & 1409 & 517 & 131 & 349 & 53 & 131 & 2833 & 2047 & 139 & 349 & 53 \\
143 & 19 & 5 & 159 & 359 & 245 & 167 & 71 & 19 & 183 & 1277 & 753 \\
184 & 3119 & 2533 & 187 & 71 & 37 & 191 & 151 & 33 & 199 & 53 & 19 \\
\hline
\end{tabular}

TABLE $4 . \tilde{\lambda}_{p}\left(\chi \omega^{k}\right)=2(3$ for the $*$-marked cases $)$

\begin{tabular}{ccc|ccc|ccc|ccc}
\hline$f_{\chi}$ & $p$ & $k$ & $f_{\chi}$ & $p$ & $k$ & $f_{\chi}$ & $p$ & $k$ & $f_{\chi}$ & $p$ & $k$ \\
\hline 7 & 3613 & 1147 & 8 & 23 & 11 & 11 & 173 & 43 & 15 & 11 & 7 \\
15 & 17681 & 5641 & 24 & 41 & 13 & 39 & 1289 & 211 & 39 & 26633 & 14233 \\
43 & 127 & 105 & 47 & 5393 & 3265 & 51 & 4261 & 2867 & 52 & 19 & 17 \\
52 & 2081 & 993 & 56 & 11 & 3 & 59 & 52313 & 44231 & 67 & 79337 & 30221 \\
67 & 263 & 217 & 71 & 89 & 43 & 79 & 307 & 299 & 83 & 677 & 169 \\
84 & 11593 & 4589 & 103 & 17 & 3 & 115 & 20357 & 16409 & 116 & 283 & 89 \\
120 & 73 & 33 & 123 & 5 & $3^{*}$ & 131 & 127 & 97 & 136 & 7 & 3 \\
136 & 4919 & 2571 & 139 & 47 & $35 *$ & 139 & 439 & 183 & 139 & 653 & 217 \\
151 & 13 & 9 & 151 & 251 & 129 & 151 & 727 & 419 & 163 & 937 & 919 \\
163 & 2441 & 1059 & 167 & 43 & 19 & 167 & 1031 & 445 & 168 & 7 & 3 \\
168 & 733 & 117 & 168 & 1361 & 205 & 184 & 5 & 3 & 187 & 103 & 87 \\
199 & 227 & 173 & & & & & & & & & \\
\hline
\end{tabular}

TABLE 5. The $\chi$-irregularity index density

\begin{tabular}{c|cc|cc}
\hline$r$ & $n_{r}$ & $n_{r}^{\prime}$ & the density & the density' \\
\hline 0 & 360567 & 360726.71 & 0.60642302 & 0.60669164 \\
1 & 180605 & 180279.99 & 0.30375222 & 0.30320562 \\
2 & 44967 & 45035.78 & 0.07562817 & 0.07574385 \\
3 & 7387 & 7499.17 & 0.01242389 & 0.01261256 \\
4 & 959 & 936.41 & 0.00161290 & 0.00157491 \\
5 & 86 & 93.53 & 0.00014463 & 0.00015730 \\
6 & 9 & 7.78 & 0.00001513 & 0.00001309 \\
7 & 0 & 0.55 & 0.00000000 & 0.00000093 \\
\hline
\end{tabular}

From these tables, we can obtain concrete information on the higher $K$-groups of the ring of integers of $\mathbf{Q}\left(\sqrt{-f_{\chi}}\right)$ (see [16, $\left.\S 4\right]$ ).

Let us call a pair of integers $(p, k)$ a $\chi$-irregular pair if $p$ is a prime, $k$ is an odd integer satisfying $3 \leq k \leq p-2, p$ divides $a_{0}\left(\chi \omega^{k}\right)=L_{p}\left(1, \chi \omega^{k}\right)$ (or $b_{0}\left(\chi \omega^{k}\right)=$ $\left.L_{p}\left(0, \chi \omega^{k}\right)\right)$, and $\left(p, \chi \omega^{k}\right)$ satisfies $(\mathrm{C})$. Further we define the $\chi$-irregularity index $r_{p}(\chi)$ by

$$
r_{p}(\chi)=\sharp\{(p, k) \mid(p, k) \text { is a } \chi \text {-irregular pair }\} .
$$




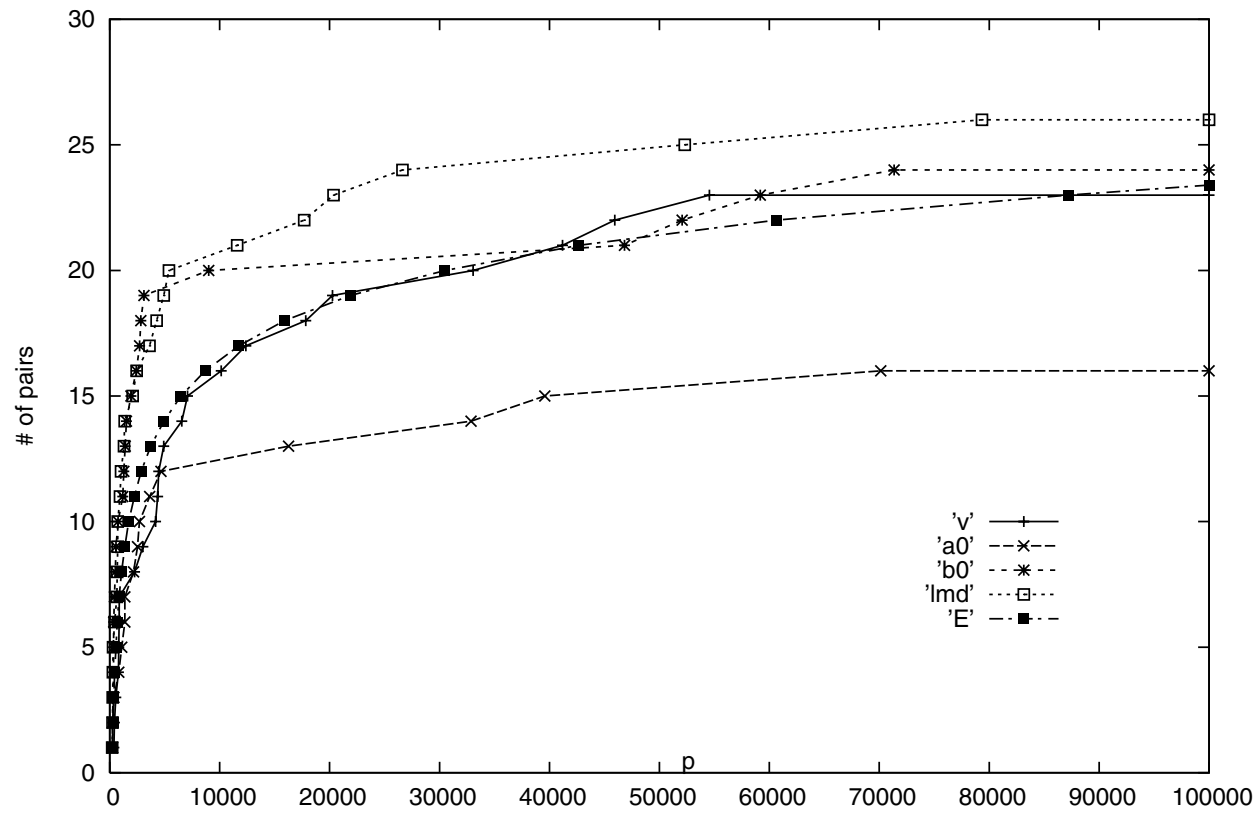

FiguRE 1. Exception pairs (odd quadratic, $1<f<200,200<$ $p<100000)$

We call a prime number $p \chi$-irregular if $r_{p}(\chi)>0$. Let $m_{p}(\chi)$ be the number of even integers $k$ with $3 \leq k \leq p-2$ such that $\left(p, \chi \omega^{k}\right)$ satisfies $(\mathrm{C})$. We define

$$
n_{r}=\sum_{(\chi, p) \text { s.t. } r_{p}(\chi)=r} 1
$$

and

$$
n_{r}^{\prime}=\sum_{\chi, p} m_{p}(\chi) C_{r}\left(\frac{1}{p}\right)^{r}\left(\frac{p-1}{p}\right)^{m_{p}(\chi)-r},
$$

where $\chi$ runs over all odd quadratic characters with $1<f_{\chi}<200$, and $p$ runs all prime numbers with $5 \leq p<100000$. The distribution of the indices of $\chi$ irregularity is given in Table 5 . The actual numbers $n_{r}$ seem to be close to the expected numbers $n_{r}^{\prime}$ (cf. [2] and [17, p. 63]).

In Figure 1, we compare the actual numbers of exceptional pairs with the expected numbers in the range $200<p<100000$. Set

$$
\begin{aligned}
& \nu(x)=\sharp\left\{\left(p, \chi \omega^{k}\right) \mid 200<p<x, \chi \omega^{k} \text { : even, } k \neq 1, \nu_{p}\left(\chi \omega^{k}\right) \geq 1\right\}, \\
& a_{0}(x)=\sharp\left\{\left(p, \chi \omega^{k}\right) \mid 200<p<x, \chi \omega^{k} \text { : even, } k \neq 1, a_{0}\left(\chi \omega^{k}\right) \geq 2\right\} \text {, } \\
& b_{0}(x)=\sharp\left\{\left(p, \chi \omega^{k}\right) \mid 200<p<x, \chi \omega^{k} \text { : even, } k \neq 1, b_{0}\left(\chi \omega^{k}\right) \geq 2\right\} \text {, } \\
& \operatorname{lmd}(x)=\sharp\left\{\left(p, \chi \omega^{k}\right) \mid 200<p<x, \chi \omega^{k} \text { : even, } k \neq 1, \tilde{\lambda}_{p}\left(\chi \omega^{k}\right) \geq 2\right\} \text {, } \\
& E(x)=\left[\sharp\{\chi\} \sum_{200<p<x, p \text { :prime }} \frac{p-3}{2} \frac{1}{p^{2}}\right],
\end{aligned}
$$

where $[*]$ is the Gauss symbol and $\chi$ runs over all odd quadratic characters with $1<f_{\chi}<200$. 


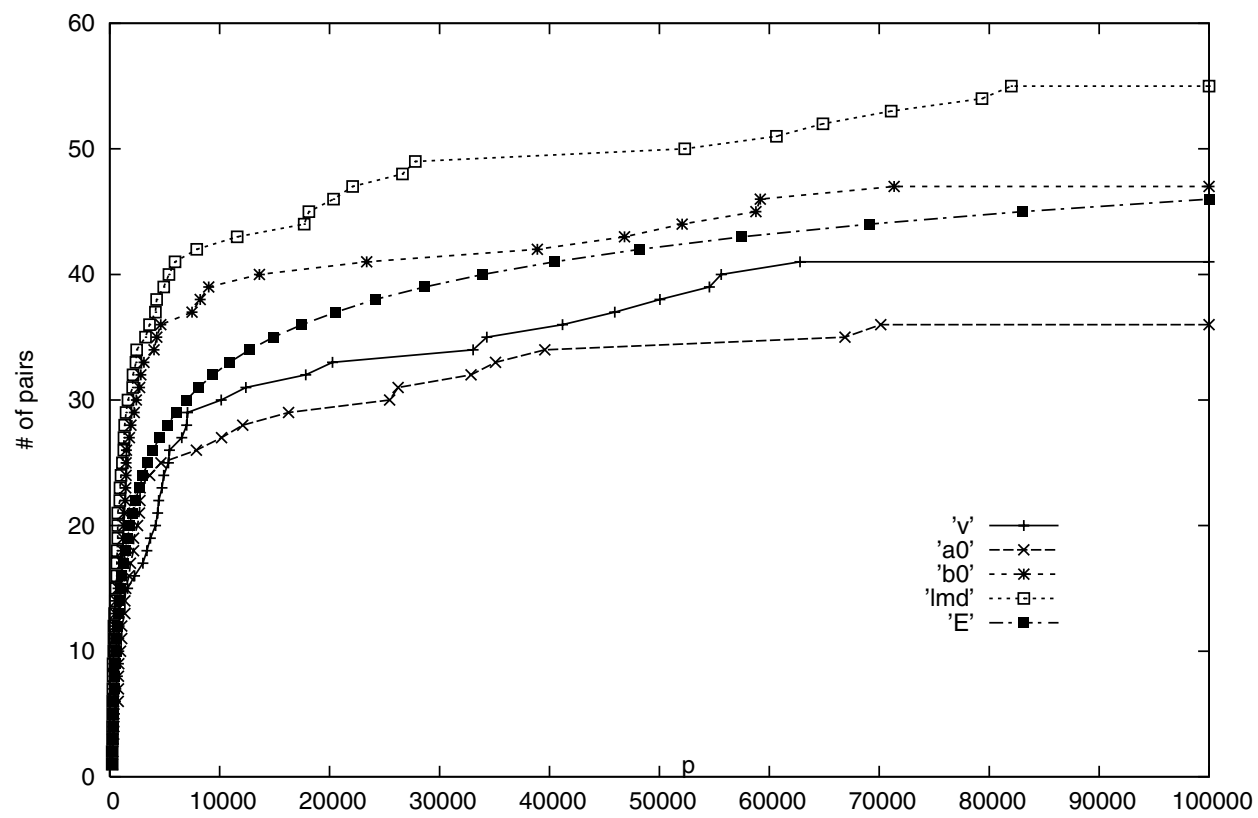

FiguRE 2. Exceptional pairs (quadratic, $1<f<200,200<p<100000$ )

In order to increase the number of samples, we combine the above data with that in [16, and obtain Figure 2. Set

$$
\begin{aligned}
& \nu(x)=\sharp\left\{\left(p, \chi \omega^{k}\right) \mid 200<p<x, \chi \omega^{k}: \text { even, } k \neq 0,1, \nu_{p}\left(\chi \omega^{k}\right) \geq 1\right\}, \\
& a_{0}(x)=\sharp\left\{\left(p, \chi \omega^{k}\right) \mid 200<p<x, \chi \omega^{k}: \text { even, } k \neq 0,1, a_{0}\left(\chi \omega^{k}\right) \geq 2\right\}, \\
& b_{0}(x)=\sharp\left\{\left(p, \chi \omega^{k}\right) \mid 200<p<x, \chi \omega^{k}: \text { even, } k \neq 0,1, b_{0}\left(\chi \omega^{k}\right) \geq 2\right\}, \\
& \operatorname{lmd}(x)=\sharp\left\{\left(p, \chi \omega^{k}\right) \mid 200<p<x, \chi \omega^{k}: \text { even, } k \neq 0,1, \tilde{\lambda}_{p}\left(\chi \omega^{k}\right) \geq 2\right\}, \\
& E(x)=\left[\sharp\{\chi\} \sum_{200<p<x, p \text { prime }} \frac{p-3}{2} \frac{1}{p^{2}}\right],
\end{aligned}
$$

where $\chi$ runs over all quadratic characters with $1<f_{\chi}<200$.

From our data, the actual numbers seem to be close to the expected numbers. Even for large $p$, it might be possible that the actual numbers are near to the expected numbers.

Finally we give an example such that $A_{n}$ is not cyclic. In [1, Aoki-Fukuda showed that

$$
A_{0}^{\chi \omega} \simeq \mathbf{Z} / p \mathbf{Z} \oplus \mathbf{Z} / p \mathbf{Z}, \quad A_{0}^{\chi \omega^{3}} \simeq\{0\}
$$

for $\left(f_{\chi}, p\right)=(4 \cdot 14606,5)$ by using cyclotomic units of $\mathbf{Q}\left(\zeta_{f_{0} l_{i}}\right)\left(l_{1}=11251\right.$ and $l_{2}=22501$ ). By our method (using cyclotomic units and Gauss sums of $\mathbf{Q}\left(\zeta_{f_{n}}\right)$ for $n \leq 2)$, we show the above and

$$
A_{n}^{\chi \omega} \simeq \mathbf{Z} / p^{2} \mathbf{Z} \oplus \mathbf{Z} / p \mathbf{Z}, \quad A_{n}^{\chi \omega^{3}} \simeq\{0\}
$$

for $n \geq 1$. First we have

$$
\left\{\begin{array}{l}
g_{\chi \omega}(T) \equiv \omega_{0}^{*}\left(T^{2}+2380 T+2025\right) \bmod p^{5}, \\
g_{\chi \omega}^{*}(T) \equiv \omega_{0}\left(T^{2}+1305 T+2150\right) \bmod p^{5},
\end{array},\left\{\begin{array}{l}
g_{\chi \omega^{3}}(T)=1 \\
g_{\chi \omega^{3}}^{*}(T)=1
\end{array}\right.\right.
$$


Hence we immediately obtain the triviality of $A_{n}^{\chi \omega^{3}}$. For $\psi=\chi \omega$, we have $\psi(p) \neq 1$ and $\psi^{*}(p)=1$.

\section{Cyclotomic units.}

$\mathbf{n}=\mathbf{0}$

$$
\begin{gathered}
\mathcal{C}_{0}^{\psi} \simeq\left(\omega_{0}, p^{2}\right) /\left(\omega_{0}\right), \\
\mathcal{E}_{0}^{\psi} \simeq\left(\mathcal{E}_{0}^{\psi}\right)^{\prime} \subseteq \Lambda /\left(\omega_{0}\right) .
\end{gathered}
$$

Hence $\left(\mathcal{E}_{0} / \mathcal{C}_{0}\right)^{\psi}$ is a subgroup of $\Lambda /\left(\omega_{0}, p^{2}\right) \simeq \mathbf{Z} / p^{2} \mathbf{Z}$.

$\mathbf{n}=\mathbf{1}$

$$
\mathcal{C}_{1}^{\psi} \simeq\left(\tilde{g}_{\psi}(T), p T, p^{3}\right) /\left(\omega_{1}\right)
$$

Let $l_{1}=1+12 f_{1} p=87636001$ and $l_{2}=1+22 f_{1} p=160666001$. By studying the image of $\mathcal{C}_{1}^{\psi}$ in $\prod_{\mathfrak{L}_{i} \mid l_{1} l_{2}}\left(\mathcal{O}_{K_{1}} / \mathfrak{L}_{i}\right)$, we have

$$
\mathcal{E}_{1}^{\psi} \simeq\left(\mathcal{E}_{1}^{\psi}\right)^{\prime} \subseteq\left(\tilde{g}_{\psi}(T), T, p\right) /\left(\omega_{1}\right) .
$$

Hence $\left(\mathcal{E}_{1} / \mathcal{C}_{1}\right)^{\psi}$ is a subgroup of $\left(\tilde{g}_{\psi}(T), T, p\right) /\left(\tilde{g}_{\psi}(T), p T, p^{3}\right) \simeq \mathbf{Z} / p^{2} \mathbf{Z} \oplus \mathbf{Z} / p \mathbf{Z}$.

$\mathbf{n}=\mathbf{2}$

$$
\mathcal{C}_{2}^{\psi} \simeq\left(\tilde{g}_{\psi}(T), p^{2} T, p^{4}\right) /\left(\omega_{2}\right)
$$

Let $l_{1}^{\prime}=1+8 f_{2} p=292120001$ and $l_{2}^{\prime}=1+14 f_{2} p=511210001$. By studying the image of $\mathcal{C}_{2}^{\psi}$ in $\prod_{\mathfrak{L}_{i}^{\prime} \mid l_{1}^{\prime} l_{2}^{\prime}}\left(\mathcal{O}_{K_{2}} / \mathfrak{L}_{i}^{\prime}\right)$, we have

$$
\mathcal{E}_{2}^{\psi} \simeq\left(\mathcal{E}_{2}^{\psi}\right)^{\prime} \subseteq\left(\tilde{g}_{\psi}(T), p T, p^{2}\right) /\left(\omega_{2}\right) .
$$

Hence $\left(\mathcal{E}_{2} / \mathcal{C}_{2}\right)^{\psi}$ is a subgroup of $\left(\tilde{g}_{\psi}(T), p T, p^{2}\right) /\left(\tilde{g}_{\psi}(T), p^{2} T, p^{4}\right) \simeq \mathbf{Z} / p^{2} \mathbf{Z} \oplus \mathbf{Z} / p \mathbf{Z}$. This implies Greenberg's conjecture for $A_{n}^{\psi}$.

By computation of Gauss sums, we will show that $\mathcal{E}_{1}^{\psi} \simeq\left(\tilde{g}_{\psi}(T), T, p\right) /\left(\omega_{1}\right)$. Hence we have $\sharp\left(\mathcal{E}_{1}^{\psi} / \mathcal{C}_{1}^{\psi}\right)=p^{3}, \sharp\left(\mathcal{E}_{2}^{\psi} / \mathcal{C}_{2}^{\psi}\right) \geq p^{3}$, and $\mathcal{E}_{2}^{\psi} \simeq\left(\tilde{g}_{\psi}(T), p T, p^{2}\right) /\left(\omega_{2}\right)$. By this isomorphism, $\operatorname{Ker}\left(A_{0} \rightarrow A_{2}\right)^{\psi} \simeq H^{1}\left(\Gamma_{0}, E_{2}\right)^{\psi} \simeq \mathbf{Z} / p \mathbf{Z} \oplus \mathbf{Z} / p \mathbf{Z}$ (see the proof of Theorem (1). Therefore we have $A_{0}^{\psi} \simeq \mathbf{Z} / p \mathbf{Z} \oplus \mathbf{Z} / p \mathbf{Z}$ and $A_{n}^{\psi} \simeq \mathbf{Z} / p^{2} \mathbf{Z} \oplus \mathbf{Z} / p \mathbf{Z}$ for $n \geq 1$ (cf. [15, Theorem1]).

Gauss sums. Since $p \omega_{0} \in\left(\tilde{g}_{\psi}^{*}(T), \omega_{1}\right)$, the exponent of $\omega_{0} X_{\infty}^{\psi^{*}} / \omega_{1} X_{\infty}^{\psi^{*}} \simeq \omega_{0} \tilde{X}_{\infty}^{\psi^{*}} /$ $\omega_{1} \tilde{X}_{\infty}^{\psi^{*}}$ is $p$. Therefore, the exponent of $\omega_{0} \tilde{A}_{1}^{\psi^{*}}$ is at most $p$. We will show that $\omega_{0} \tilde{A}_{1}^{\psi^{*}} \simeq \mathbf{Z} / p \mathbf{Z} \oplus \mathbf{Z} / p \mathbf{Z}$ by using Gauss sums and prime numbers.

Set $h(T)=21 T^{4}+17 T^{3}+9 T^{2}+5 T+15$. Then we have

$$
h(T) g_{\psi}^{*}(T) \equiv p \omega_{0} \bmod \left(\omega_{1}, p^{2}\right) .
$$

Let $\mathbf{e}_{\psi^{*}, m} \in \mathbf{Z}[\Delta]$ such that $\mathbf{e}_{\psi^{*}, m} \equiv e_{\psi^{*}} \bmod p^{m}$. For $\mathfrak{L}_{i} \mid l_{1} l_{2}$, let $g_{1}\left(\mathfrak{L}_{i}\right)$ be the Gauss sum of $K_{1}$ which satisfies

$$
\left(g_{1}\left(\mathfrak{L}_{i}\right)^{\mathbf{e}_{\psi^{*}, m}}\right)=\mathfrak{L}_{i}^{f_{\chi} \theta_{1} \mathbf{e}_{\psi^{*}, m}},
$$

where $\theta_{1} \in \mathbf{Q}\left[\operatorname{Gal}\left(K_{1} / \mathbf{Q}\right)\right]$ is the Stickelberger element (see [7, pp. 42-45] for details). Hence for any integer $m \geq 1$, there exists $g_{m}^{\prime} \in K_{1}$ such that

$$
\left(g_{1}\left(\mathfrak{L}_{i}\right)^{\mathbf{e}_{\psi^{*}, 1}}\right)=\mathfrak{L}_{i}^{f_{\chi} \theta_{1} \mathbf{e}_{\psi^{*}, m}}\left(g_{m}^{\prime}{ }^{p}\right) .
$$

Since $G_{\psi}^{*}(T) \equiv \mathbf{e}_{\psi^{*}, m} \theta_{1} \bmod \left(p^{m}, \omega_{1}\right)$, we have

$$
\left(g_{1}\left(\mathfrak{L}_{i}\right)^{\mathbf{e}_{\psi^{*}, 1} h(T)}\right)=\mathfrak{L}_{i}^{\omega_{0} p u(T) \mathbf{e}_{\psi^{*}, m}} \mathfrak{L}_{i}^{p^{m} v \mathbf{e}_{\psi^{*}, m}}\left(g_{m}^{\prime}{ }^{p h(T)}\right)
$$


for $u(T) \in \Lambda^{\times}$and $v \in \mathbf{Z}_{p}\left[\operatorname{Gal}\left(K_{1} / \mathbf{Q}\right)\right]$. Let $l_{1}=1+11 f_{1}=16066601, l_{2}=$ $1+14 f_{1}=20448401, l_{1}^{*}=1+4\left(2 f_{1} l_{1} l_{2}\right)=3838880957714195684801$ and $l_{2}^{*}=1+$ $7\left(2 f_{1} l_{1} l_{2}\right)=6718041675999842448401$. By studying the images of $g_{1}\left(\mathfrak{L}_{i}\right)^{\mathbf{e}_{\psi^{*}, 1} h(T)}$

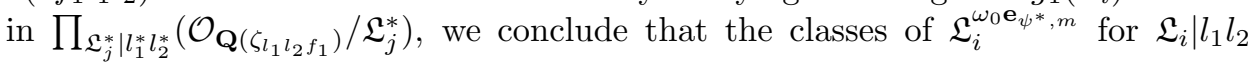
generate a subgroup of $\tilde{A}_{1}^{\psi^{*}}$ whose quotient is isomorphic to $\mathbf{Z} / p \mathbf{Z} \oplus \mathbf{Z} / p \mathbf{Z}$. Since $\sharp\left(\omega_{0} \tilde{X}_{\infty}^{\psi^{*}} / \omega_{1} \tilde{X}_{\infty}^{\psi^{*}}\right)=p^{2}$, this happens only when $\nu_{1,0} \tilde{Y}_{\infty}^{\psi^{*}}=\omega_{1} \tilde{X}_{\infty}^{\psi^{*}}$, i.e. $\tilde{Y}_{\infty}^{\psi^{*}}=$ $\omega_{0} \tilde{X}_{\infty}^{\psi^{*}}$. By Lemma $3\left(X=\tilde{X}_{\infty}^{\psi^{*}}\right.$ and $\left.Z=\omega_{1} \tilde{X}_{\infty}^{\psi^{*}}\right)$ and the class field theory, we have $\left\langle\tilde{\sigma}_{\mathfrak{L}_{i}, m}^{\mathbf{e}_{\psi^{*}, m}}\right\rangle_{\mathfrak{L}_{i} \mid l_{1} l_{2}}=\tilde{X}_{1}^{\psi^{*}}$. By Lemma $4\left(n=n^{\prime}=1\right)$ and the image of $\mathcal{C}_{1}^{\psi}$ in $\prod_{\mathfrak{L}_{i} \mid l_{1} l_{2}}\left(\mathcal{O}_{K_{1}} / \mathfrak{L}_{i}\right)$, we obtain $\mathcal{E}_{1}^{\psi} \simeq\left(\tilde{g}_{\psi}(T), T, p\right) /\left(\omega_{1}\right)$.

We used thirty personal computers for three months to make the tables in this section. The programs were written in UBASIC and C, in which the GNU MP library was included. For the last example, it took a few minutes to calculate cyclotomic units modulo prime ideals, and thirty minutes to calculate Gauss sums modulo prime ideals on one PC (CPU: Pentium IV, 3.6GHz, RAM 2GB). In [1], it took 6 hours and 42 minutes to compute $A_{0}$ by using Alpha 21264, 667MHz, RAM 4GB.

\section{REFERENCES}

1. M. Aoki and T. Fukuda, An algorithm for computing p-class groups of abelian number fields, Lecture Notes in Comput. Sci. 4076, Springer, Berlin, 2006, pp. 56-71.

2. J. Buhler, R. Crandall, R. Ernvall, T. Metsänkylä, and A. M. Shokrollahi, Irregular primes and cyclotomic invariants to 12 million, J. Symbolic Comput. 31 (2001), 89-96. MR.1806208 (2001m:11220)

3. B. Ferrero and R. Greenberg, On the behavior of $p$-adic L-functions at $s=0$, Invent. Math. 50 (1978), 91-102. MR0516606 (80f:12016)

4. B. Ferrero and L. Washington, The Iwasawa invariant $\mu_{p}$ vanishes for abelian number fields, Ann. of Math. 109 (1979), 377-395. MR0528968 (81a:12005)

5. R. Gillard, Remarques sur les unités cyclotomiques et les unités elliptiques, J. Number Theory 11 (1979), 21-48. MR0527759 (80j:12004)

6. R. Greenberg, On the Iwasawa invariants of totally real number fields, Amer. J. Math. 98 (1976), 263-284. MR0401702 (53:5529)

7. H. Ichimura, Local units modulo Gauss sums, J. Number Theory 68 (1998), 36-56. MR:1492887 (98k:11154)

8. H. Ichimura and H. Sumida, On the Iwasawa invariants of certain real abelian fields II, Internat. J. Math. 7 (1996), 721-744. MR.1417782(98e:11128c)

9. K. Iwasawa, Lectures on p-adic L-functions, Ann. of Math. Stud., vol. 74, Princeton Univ. Press: Princeton, N.J., 1972. MR.0360526 (50:12974)

10. MR0349627 (50:2120)

11. J. S. Kraft and R. Schoof, Computing Iwasawa modules of real quadratic number fields, Compositio Math. 97 (1995), 135-155. MR1355121 (97b:11129)

12. T. Kubota and H.W. Leopoldt, Eine p-adische Theorie der Zetawerte, I. Einführung der p-adischen Dirichletschen L-Funktionen, J. Reine Angew. Math. 214/215 (1964), 328-339. MR.163900 (29:1199)

13. B. Mazur and A. Wiles, Class fields of abelian extensions of Q, Invent. Math. 76 (1984), 179-330. MR0742853 (85m:11069)

14. M. Ozaki, On the cyclotomic unit group and the ideal class group of a real abelian number field. I, II, J. Number Theory 64 (1997), 211-222, 223-232. MR.1453211 (98c:11121)

15. H. Sumida-Takahashi, Computation of Iwasawa invariants of certain real abelian fields, J. Number Theory 105 (2004), 235-250. MR2040156 (2005a:11171) 
16. - The Iwasawa invariants and the higher K-groups associated to real quadratic fields, Exp. Math. 14 (2005), 307-316. MR2172709 (2006j:11152)

17. L. Washington, Introduction to cyclotomic fields. second edition, Graduate Texts in Math., vol. 83, Springer-Verlag: New York, 1997. MR1421575 (97h:11130)

Faculty and School of Engineering, The University of Tokushima, 2-1 MinamijosanJIMA-CHO, TOKUSHIMA 770-8506, JAPAN

E-mail address: hiroki@pm.tokushima-u.ac.jp 